Proceedings of the 2011 Winter Simulation Conference

S. Jain, R.R. Creasey, J. Himmelspach, K.P. White, and M. Fu, eds.

\title{
TUTORIAL ON THE SIMULATION OF HEALTHCARE SYSTEMS
}

\author{
Stephen D. Roberts \\ North Carolina State University \\ Edward P. Fitts Department of Industrial and Systems Engineering \\ 378 Daniels Hall \\ Raleigh, NC 27695
}

\begin{abstract}
For a variety of reasons, simulation has enjoyed widespread application in health care and health care delivery systems. Although the dominant modeling methodology is discrete event simulation, numerous studies employ system dynamics, agent-based simulation, and hybrid/combined methods. Software has been increasingly adapted to health care through enhanced visualizations and modeling. Virtually every health care environment has been studied using simulation including hospitals, extended care, rehabilitation, specialty care, long-term care, public health, among others. Frequent problems are patient flow, staffing, works schedules, facilities capacity and design, admissions/scheduling, appointments, logistics, and planning. Health care problems are especially complicated by the fact that "people serve people," meaning people are both the customer and the supply. The customers arrive through a complex decision process that produces uncertain demand. The response is an even more complex organization of health care resources, each of which play a distinctive and overlapping role, providing a unique simulation challenge.
\end{abstract}

\section{INTRODUCTION}

Simulation of health care systems is about the improvement of health care. Health care systems performance may be generally measured in terms of its access, costs, and quality. A simulation of an emergency department that yields fewer people who "leave without being seen (LWBS)" improves access to that emergency department by allowing more people to be seen. A simulation of an emergency department that reduces waiting time experienced by the patient improves the quality of care that the system delivers to the patient simply by attending more efficiently to that patient. A simulation of an emergency department that uses the staff more effectively (efficiently) also reduces the cost of the care delivered since those costs are now spread over more patients. If that simulation is able to improve access without increasing costs or lowering quality, or if the simulation can lower costs without reducing access or lowering quality, or if that simulation can increase quality without reducing access or increasing cost, then those simulation results "break" the iron triangle of health services.

The iron triangle is the belief by some that access, costs, and quality are so tightly bound that a positive change in one measure of performance is obtained only by a negative change in one or both of the other measures. For example, costs cannot be lowered without reducing access and/or reducing quality. Therefore one of the primary goals of a health systems simulation (and any other performance improvement methods) should be to break that iron triangle among access, costs, and quality and to improve health care. Concern with only one measure of effectiveness, say quality of care, does injustice to concern with the system of care.

The emergency department example is a microcosm of the larger problems faced in health care relative to access, costs, and quality. In the United States, the cost of health care exceeds $19 \%$ of the gross 


\section{Roberts}

national product; more than double that of one decade ago. Government spending on health care for Medicare and Medicaid threatens to bankrupt government services. Spending through health insurance has risen to the point that many companies and firms are threatening to remove health insurance as a benefit and/or force employees to bear a much larger burden of the costs. The point is that health care costs seem destined to rise and to remain a significant problem for decades to come. Policy makers and politicians debate and argue the various merits of "system" changes. No doubt the debate will be expanded during forthcoming elections in the United States. While the problems of health care seem particularly acute within the US health care enterprise, many delivery problems plague health care systems in other countries.

In the US, health care is primarily composed of "silos" of care where professional boundaries, namely the practices of doctors, nurses, pharmacists, technicians, etc. determine the extent of influence. Individual health care institutions compete for the same patients and physicians. These silos are well established for the providers, but patients must navigate through them for comprehensive care. Simulation is a tool that tends to examine the health care delivery system and to cross those traditional boundaries.

The challenge to improve health care is both acute and persistent. Because health care is large and complex, there are many approaches to improvement and many issues to confront. Simulation can be viewed as only one approach, popular in the community who knows about it and largely unknown otherwise in health care. Thus the opportunity exists for more people in health care to learn about simulation. The ability of simulation to provide both high-level strategic (system-wide) value as well as a tool for operational performance improvement creates a vehicle for widespread use of simulation in the improvement of health care.

Of course, the merit of the system perspective also encounters a void of authority. The "decisionmaker" for the system is rarely a single person or even a single organizational structure, but instead, the simulation must often be "sold" to a multi-disciplinary audience, each of whom may veto a recommended change. Hence it is often the case that a simulation is needs to be embedded into a general process improvement program that addresses cost, access, and quality.

As an introduction to the simulation of healthcare systems, this paper will focus on (1) the domains of healthcare simulations, (2) healthcare simulation tools, (4) what makes simulation attractive in health care, and (4) fundamental issues in building healthcare simulations. It is assumed that the reader has some previous exposure to doing simulation. Prior literature reviews of health care simulation include the works of England and Roberts (1978), Klein et al. (1993), Wilson (1981), Jun, Jacobson, and Swisher (1999) which was updated in Jacobson, Hall, and Swisher (2006), Fone et al. (2003), and Brailsford et al. (2009). Prior tutorials on the simulation of healthcare systems include Lowery (1996), Lowery (1998) and Standridge (1999).

\section{THE DOMAIN OF HEALTHCARE SIMULATIONS}

There has been literally thousands of health care simulation articles published throughout the literature. The 1978 survey by England and Roberts (1978) cited over 1200 models. Certainly since that time, the literature has grown by at least twice that. The review by Jacobson, Hall, and Swisher (2006) has over 175 references, while the review by Brailsford et al. (2009) included 342 modeling references with the majority being simulation.

Two of the reviews included general modeling approaches in addition to simulation. The review by Fone et al. (2003) concentrated on the use and value of simulation to population health and health care delivery, reviewing about 182 papers. The review by Brailsford et al. (2009) examined the research literature on simulation and modeling to identify the range of modeling approaches, the domains of application, and level of implementation. More recent articles on health care simulation are found in Health Care Management Science, the IIE Transactions on Healthcare Systems Engineering, and Medical Decision Making, among others.

Based on their review of the literature England and Roberts offered a taxonomy for the use of computer simulation in health care. A revision of that taxonomy would be: 
1. Hospital Systems models including admissions control, bed allocation and planning, nurse staffing, materials handling and logistics, specialty hospitals such as nursing homes, inpatient rehabilitation care, cancer care

2. Hospital Departmental models including ambulance and emergency service, laboratory, radiology, surgery and recovery, pharmacy, supply and support

3. Ambulatory Care models including primary and specialty outpatient clinics and doctors' offices, appointment scheduling, staffing patterns, room layout and design, flow control, patient panel size

4. Other Ambulatory Care such as home care, dental practice, public health and disease control, mental health, drug recovery and rehabilitation

5. Planning models for people such as professions' planning and forecasting, skills substitution and staffing

6. Health Care Systems Planning such as Certificate of Need (CON), community and regional health care, health maintenance organizations and prepayment, managed care

7. Other Health Care models including blood banking, transplant management, patient education centers, telemedicine

8. Medical Decision making such as screening, treatment choice, cost-effectiveness, organ transplantation

This taxonomy continues to be relevant to newer simulation studies. The more recent review by Jacobson et al. (2006) uses some refined categories:

1. Patient Flow Optimization and Analysis

a. Outpatient Scheduling

b. Inpatient Scheduling and Admissions

c. Emergency Room Simulation Models

d. Specialists Clinics

e. Physician and Health Care Staff Scheduling

2. Health Care Asset Allocation
a. Bed Sizing and Planning
b. Room Sizing and Planning
c. Staff Sizing and Planning

Clearly simulation has a widespread and comprehensive application in health care. Overwhelmingly the applications are to operational problems. However, there are a number of applications to medical decision making, policy, and planning (Klein et al. 1993; Fone et al. 2003; Brailsford et al. 2009). The growth of simulation in health care is noted by almost all who have studied the literature.

\section{HEALTHCARE SIMULATION TOOLS}

Most healthcare simulations are done using a simulation language. In the past, general purpose simulation languages have dominated the applications. Popular choices (in no particular order) have been the discrete event languages such as GPSS/H (WolverineSoftware 2011a), Arena (RockwellSoftware 2011b), ExtendSim (ImagineThat!Inc. 2011), ProModel (ProModelCorporation 2011a), Simul8 (Simul8Corporation 2011b), Witness (Lanner 2011), SLX (WolverineSoftware 2011b), Simio (SimioLLC 2011b), and AutoMod (AppliedMaterialsInc. 2011). Many of these same simulation vendors have special sections of their websites devoted to the healthcare industry, which provide white papers and more description of the capabilities of each respective software for modeling healthcare systems. For example, view the sites for Arena (RockwellAutomation 2011a), Simul8 (Simul8Corporation 2011a), and Simio (SimioLLC 2011a). In recent years, some healthcare simulation languages/products have been devel- 


\section{Roberts}

oped, including MedModel (ProModelCorporation 2011b) and FlexSim HC (FlexsimSoftwareProducts 2011). These healthcare simulation products have built-in health care objects such as patients, doctors, beds, and so forth. Furthermore, their animation libraries contain animation components found in exam rooms, laboratories, pharmacies, etc. The success of these healthcare targeted simulation products, when considered with the growth of simulation in healthcare, suggests that more simulation vendors will be offering healthcare simulation products.

Because implementation dominates the application of healthcare concerns, visualization of the simulation model often plays a key role in whether the simulation is accepted by a diverse set of stakeholders. Whether it's justified or not, people in healthcare tend to judge the quality of the simulation by the quality of the animation. How to judge the quality of the animation is clearly in the eye of the judge, but in general, the more realism the stakeholder can "see" the more that person is likely to accept the results of the simulation. While it is possible to have a successful simulation without animation, a good animation remains an important "sales" tool to involve and win-over the various healthcare interests. For these reasons coupled with better computing power, software developers are following suit by including high fidelity, three-dimensional animation capabilities.

\subsection{Simulation Modeling Methods and Approaches}

There are a number of ways to characterize simulation tools using the approach taken. Except for a few simulation tools specific to healthcare, the general characterizations apply. Traditionally, simulation includes Monte Carlo simulation (random sampling), discrete-event simulation (next-event), continuous simulation (continuous change variables), combined simulation (combination of discrete-event and continuous), system dynamics (an adaptation of continuous simulation), and agent-based simulation (objectoriented simulation). All these are found in the health care simulation literature. Discrete-event simulation tends to dominate health operations problems due to the need to include waiting when demand for a service exceeds its capacity. Monte Carlo simulation models, such as those built from Crystal Ball (Oracle 2011) or@Risk (PalisadeCorporation 2011), are used for medical decision making and for aggregate health planning. Continuous models in health care are mostly of the systems dynamics form and are used in health policy and planning. Older compartmental, differential equation-based models have been supplanted by agent-based simulations in epidemiology and infection control to model contact networks and capture interactions among individuals.

Although simulation is basically an experimental tool, its combination with optimization is increasing, especially in a research context. Several of the commercial simulation languages now have optimization components, which can be used to find near-optimal solutions to explicit objective functions. Typically, the simulation determines the objective while the optimization guides the specification of variable values. A few health care studies employ recursive simulation in which the simulation and the optimization are separate processes which communicate with each other. The combination of simulation and optimization holds promise for solving some multi-objective problems (minimizing waiting time while maximizing utilization). Nevertheless the need to provide an explicit objective function is extremely challenging in the healthcare setting where statement of a goal is so controversial and the system being modeled is complex.

\section{WHAT MAKES SIMULATION ATTRACTIVE IN HEALTH CARE?}

Sometimes the value of simulation in healthcare is overlooked or forgotten. Here are some qualities of simulation that make it especially attractive in health care systems.

- Variability: Very little in health care is certain. In fact, it would be more accurate to claim that everything (well, almost everything) is stochastic. Simulation can incorporate variability through its handling of random variables and probabilistic outcomes. 
- Complexity: Health care is a complex system of human behavior. Doctors, nurses, pharmacists, and technicians all interact to provide patient care. And the patient is often left to negotiate the maze of health care options, all the way from finding out where the lab is in the hospital to deciding whether their loved one is to receive that operation. Human behavior defies causal modeling. Nonetheless simulation can provide descriptive models of what transpires. It is a level of complexity that is often beyond the flow in a network of queues (although a network of queues may be a good starting point). Simulation modelers can build models of the system they see, as opposed to the system someone idealizes.

- Assumptions: All modeling tools make certain assumptions about the real system. It may be that the arrivals are Markov or that the objective function is linear. Simulation has the advantage of requiring fewer assumptions by the modeler. Thus the modeler is free to model the system as it exists rather than modeling it in an idealized form. However, all models have assumptions, so it is incumbent on the modeler to be clear about the assumptions, especially since health care is so sensitive to behavioral issues. Ignoring the assumptions about actual behavior can invalidate an otherwise great simulation.

- Ease of Use: Ease of use contributes to the application of simulation. Although the use of simulation requires that the modeler work closely with the stakeholders and develop a simulation that is acceptable to a variety of perspectives, the ease with which models can be built is a tribute to the value of simulation languages. While knowing one simulation language is generally sufficient, knowing and using more than one simulation language enhances the modeling alternatives.

- What if: One of the most attractive features of simulation is its experimental nature. People in health care like experiments and understand them. Experiments with the simulation model are a natural approach to the use of simulation. It also gives different people a chance to inject their ideas about how the system might be improved. This "what-if" analysis often inspires a full discussion of trade-offs among objectives.

- Perspectives: If the modeler is successful in providing a useful simulation language, the experience and results will bring a new perspective to the organization. In particular, health care workers, who usually have no prior simulation exposure, often begin to see how patient flow, resource use, scheduling and other relationships affect the quality of patient care. This "systems perspective" introduces a broader concern with potential changes and can enhance decision-making by the stakeholders. This change in perspective may trump the actual benefits of the simulation results.

- Acceptance: Simulation models tend to be accepted in the health care environment because they often provide a visual interpretation on the model, they make few assumptions about the real system, and can provide experimental results. If the various stakeholders are incorporated into the model-building process, the acceptability is further enhanced.

- Lean/Six Sigma: Simulation is increasingly seen as a tool that enhances a lean/six sigma program in health care. Incorporating simulation into a general performance improvement program enhances the performance improvement potential and provides an opportunity to address many health care delivery issues not captured by a simulation.

\section{FUNDAMENTAL ISSUES IN BUILDING A HEALTHCARE SIMULATION}

\subsection{Barriers to Implementation}

In spite of the growth in the application of simulation to healthcare, there remain certain inescapable issues that seem to be discovered and re-discovered. The most prominent concern is with the implementation on healthcare simulations. There are many factors that contribute to this consistent problem. 


\subsubsection{The Decision-Making Structure in Health Care}

Health care is organized about professional disciplines. Doctors do doctoring, nurses do nursing, pharmacists handle drugs, lab tech process lab specimens, etc. A comprehensive simulation must satisfy a number of interested (and not so interested) parties. Decisions are made by consensus, not through authority. One unhappy party can scuttle an entire project. It is also important to note that not all stakeholders are equal. Few changes in health care delivery can be achieved without the implicit, if not explicit, approval of the medical staff. In spite of a growing sharing of authority in health care, doctors are often the final authority and model implementation needs to pay special attention to their reaction.

\subsection{2 "NIH: Not Invented Here"}

Simulations at one location never appear "portable" to another site. Instead, each location must create its own simulation, meaning that the whole modeling effort must be replicated. Data, systems, results, and so forth seem to be only relevant if collected at this site. Naturally the simulation literature is full of simulations that focus on the same problems, such as emergency room flow. Implementation therefore is rarely supported by success at other health care sites or institutions. However, being ignorant of what has been done is no excuse for a poorly done simulation model.

\subsubsection{Simulation Models are Personal}

Simulation models can be made arbitrarily complex. There is usually no agreement on the "level of detail" sufficient to be included in the model. Furthermore what is included is often based on the skill and perspective of the modeler. For example, an outpatient appointment system may include early/late arrivals and "no-shows." But these characteristics may or may not be a function of the time of day, the urgency of the appointment, the appointment reminder systems in place, and the behavior/attitude of the patients. Individual simulation modelers may have surprisingly different models for the same problem, so that implementation may be a confusion of viewpoints.

\subsubsection{Multiple Goals and Stakeholder Interests}

The fact that there are different health care system goals such as cost, access, and quality naturally produce confusion as to which goal is most important or which goals are achieved. Usually, a concentration on one outcome, such as reducing delay in patient flow, leaves open the concerns with quality and cost. Because different health care stakeholders have different frames of reference and different points of view, the simulation model results may be lost among the various interests.

\subsubsection{Not the Important Problem}

One of the most insidious problems that face the simulation modeler is the potential over-use (misuse) of simulation. Simulation is a convenient modeling tool with the capacity to model complex systems. Yet simulation remains only a tool and not a solution. So it is not uncommon to see a simulation used when a simple spreadsheet or even a set of simple calculations would suffice. The first step in deciding to use a simulation is to determine that a simulation is needed. It's hard enough to do a simulation, but to do one when it's not required is a waste of time. Remember that the third statistical error is "solving the wrong problem."

\subsubsection{Educate the stakeholder}

The oft-heard recommendation to involve the stakeholders early in the simulation study is truer in health care systems than in other areas of application. Generally, the healthcare stakeholders have no prior 
knowledge of simulation and must be educated as to its potential use. If this education doesn't begin at the genesis of simulation study, then the simulation modeler risks a substantial loss of interest in the final results and recommendations.

\subsubsection{Lack of Validation}

One of the most difficult steps in a simulation project is validation, namely making sure that the model reflects the essence of the real-world being modeled. Validation is especially critical in health care, since input data is often hard to obtain in a health care setting and assumptions can make the model invalid.

\subsection{Building and Using a Healthcare simulation}

The steps in a general simulation study include: (1) defining the problem from analysis of the real system, (2) construction of a conceptual model, (3) creation of the computer simulation model, (4) obtaining relevant results and experimentation, and (5) applying the results in decision-making. Of course many of these steps have substantial details. In the construction of the model, appropriate model input such as statistical representations must be developed. This development requires data and data analysis. Validation should persist throughout the study from conceptual validation through operational validation to the establishment of credibility. Likewise careful attention must be paid to verification that the model performs as we intend it to and that it produces "correct" results.

Many of the fundamental issues in building a healthcare simulation are the same as those in other simulation applications. In health care simulations, not unlike other fields, special attention needs to be given to working closely with the stakeholders from conceptualization to realization. A detailed and credible animation will greatly ease communication and help establish confidence in the model. It is important to solve the right problem and not simply apply simulation. Review the prior simulation work that has been published and disseminated, and be alert to the pitfalls that surround those who misunderstand the nature of the health care environment.

\subsection{Guidance on Simulation Modeling of Healthcare}

Below are some specific issues that should be understood and characteristics of healthcare simulation that at times differ greatly from systems in other industries.

\subsubsection{The Central Role of People}

A constant theme in health services is that it is composed of people (doctors, nurses, etc.) serving people (patients). This service also extends to representations of people, such as their medical records, their prescriptions, their laboratory specimens, their x-rays, and so forth. But the main service is to the patient. People objects are not inanimate but sentient. They make decisions and respond to needs. In other words, they are not necessarily systematic in providing or receiving care. They have human behavior which affects how the delivery system functions. This realization will thread itself in almost every healthcare simulation. Yet labor is at the heart of almost all health care operations and the organization and management of the labor is central to the health care function. Improving the use of labor while maintaining the other issues of quality and access can be a significant contribution of simulation. If the simulation leads to a better understanding of labor use and the standardization of that use, then the simulation study will be a success, regardless of its outcomes.

\subsubsection{The Data Challenge}

In spite of its need to report various health outcomes to government and insurance organizations, there is very little data on the operational aspects of health care. Even data on simple questions like how does radiology schedule its patients, what is the length of stay in a Post-Anesthesia Recovery Area (PACU), and 
how many nurses were used in a medical floor have unbelievable complicated answers. Getting data to "fit" a statistical distribution on the amount of time a doctor spends seeing a patient in an outpatient clinic will likely be non-existent. Therefore, it is likely a simulation modeler in health care will engage in an extensive data collection effort as a part of a simulation model development. Because so much data will need to be collected, a "rough-cut" model is needed before engaging the detailed data collection, so that only important data is collected.

\subsubsection{Rough-Cut Data in Health Care}

A rough-cut model developed early in the simulation life cycle can help to focus the simulation project and to avoid intrusive data collection of unnecessary data. Rough-cut simulations play an especially important role in health care since data is often such a challenge. To simplify the effort, the modeler can solicit from the various members of the organization the data needed in the form of estimates. The easiest is to ask for a minimum, a mode, and a maximum (a mode or most likely value is preferred to a mean, since a mean is a computed value). These estimates can be converted into a Pert or Beta distribution for input to the simulation. Generally speaking, a simulation based on such information usually performs quite well and can give early results to engage stakeholders.

\subsubsection{Health Care Operational Data}

Operational data is most useful if it is used for more than simulation input. For instance, if the operation data can be part of a decision support system, say for benchmarking, then the data has a permanent use and its accuracy will be maintained. Direct observation via time study is usually the most reliable, although it can become intrusive if not introduced properly. Other technology such as time clocks and RFID tags (interior GPS) can also be used, if the investment can be justified. Such justification is enlarged if the use of the data can augment the operational informatics in the health institution.

\subsubsection{Limits on Cross-Training}

A fairly standard and effective approach to the utilization of people in a service organization is through the use of cross-training. Cross-training allows people to share tasks so that the workload can be evenly distributed. Cross-training has potential in health care, however there are significant limitations. First, there are extensive professional boundaries. Nurses can't do what doctors do. Technicians can't do what nurses do. Thus in a busy outpatient clinic, nurses can't simply substitute for doctors. The same observation can be made in other health care settings. These professional boundaries place a limit on the amount of task-sharing. However if these limits can be breached, then significant gains in workload distribution can be obtained. A further complication of the processing time exists when substitution occurs. For instance if a clerk is used to collect vitals and place a patient in an exam room when the nurse is busy, is the processing time identical? Often it is not and substitutes have a different processing time, which if ignored, can lead to misleading conclusions regarding productivity of cross-training.

\subsubsection{Patient Arrivals}

Traditional simulations often feature random arrivals, yet in health care, with the exception of emergency arrivals, most arrivals are scheduled. Patients are typically given appointments both in inpatient and in outpatient settings. In the case of scheduled arrivals, patient behavior relative to those arrivals become relevant. In the outpatient or ambulatory settings, patient appointments serve to insure that patients available exceed the capacity available, at least at the beginning of the session. Patients, on the other hand, have varying arrival patterns, most arriving early for their appointments, some arriving late, some not even showing up, and some "walking-in". The degree to which each of these behaviors apply depends on the type of appointment, the nature of the patient population, and random unknown factors. Nevertheless, 
the simulation model should account for these if the patient arrival process is accurate. It should be noted, parenthetically, that many commercial simulation do not easily accommodate scheduled arrivals since arrivals are typically bootstrapped on the prior arrival, but in health care, an early arriving patient for the next appointment may be ahead of a late arriving patient for this appointment. Finally, it should be noted that random arrivals in a health care facility (such as an emergency department), will not be timehomogeneous. Instead, the arrival process will change depending on the time of day, the day of the week, and sometimes the month.

\subsubsection{Patient Scheduling and Appointments}

Determining a method of giving patients appointments has been and remains an active research issue. There are few widely accepted scheduling methods. Most are some form of a block scheduling system in which blocks of patients are given arrival times. The blocks insure against no-shows and accommodate walk-ins. Nevertheless, there remains a risk that staff may be idle due to the lack of patients and the period of work (such as a morning or afternoon) may be unduly delayed resulting in overtime. In scheduling surgery, the schedule is greatly complicated by the nature of the surgery, the preparation of the operating room (OR), the presence of staff (including anesthesia), and the availability of recovery (PACU) space.

\subsubsection{Health Care Capacity Modeling}

Instead of attempting to include the arrival process in a health care simulation, it is sometimes better to model system capacity, assuming that the appointment system will provide patients to the health care operation. By assuming patients are available as needed, a system capacity can be simulated. While such as capacity would probably never be achieved, it can be used as a benchmark against which to evaluate alternative system proposals. Furthermore, it avoids the knotty problem of modeling the arrival process.

\subsubsection{Team Care}

The increasing use of Team Care for many health care settings, both inpatient and outpatient, provides a significant simulation modeling challenge. Essentially team care means that multiple "resource types" are being used in the system of care, some being used simultaneously. Thus the simulation model needs to be able to accommodate some complex resource requirements. For instance, a particular patient evaluation may require an attending physician, a nurse, a social worker, and a resident. It may be the case that this team is composed of only specific persons or it may be composed of types (such as any social worker is acceptable). Also, as with cross-training, the processing time of team care can become an issue if the size and composition of the team varies. Once again, ignoring this important impact can result in a wrong evaluation of the team care benefits relative to productivity.

\subsubsection{Continuity of Care}

Continuity of care, namely the constant association of a provider with a patient is sometimes viewed as a desirable characteristic, especially in nursing. The nurse that gathered your vital signs and placed you in the exam room should be the same nurse that assists you at the end of the exam. The nurse that attends to you in a ward should be the same nurse you see on the following day. The growing use of the "Hospitalist" is recognition of the importance of continuity of medical care. Of course, staffing limitations and schedules don't always permit a continuity of care, but in those cases where it is desirable, the simulation model should be able to "remember" the initial assignment of resources and be able to recall that same assignment later. 


\subsubsection{Synchronizing Patient Entities}

Although patients are often the central entity of interest, the patient alone is rarely the only entity in the system of care. Patients have medical records, prescriptions, lab specimens, and x-rays which also characterize them. These entities are derived from the patient and affect the flow of patients because patients must wait for the ancillary services to be complete before they can proceed. This patient waiting is not due to an immediate resource limitation but due to the completion of a related process. In other words the related entities must be synchronized and a simulation model of this system will need to be able to link all these entities together. Note that this linking is not like the linking of products in a customer order because the entities are first derived from the patient and then synchronized (not assembled) with the patient.

\subsubsection{Multi-objective Performance Criteria}

It is difficult to easily define the general performance characteristics in health care. They often depend on the individual institution or decision. Most operational simulations provide information about queuing, cycle times, and resource utilization. In health care the cycle times and queuing may need to be subdivided according to patient acuity or patient type. Resource utilization may need to be obtained for individuals as well as groups. In health care, space is often a critical resource. Exam rooms, recovery beds, ward beds, operating rooms, etc. all require special attention and most often need to be included in the simulation model. Layout of space is a complicating factor and changes in layout are quite difficult for existing facilities.

\subsubsection{Diversity of Stakeholder Interests}

The diversity of stakeholder interests brings an important dimension to health care simulations and health care delivery studies in general. This unique feature of health means that each simulation crosses professional silos and each category of people included must be considered. For example, suppose you are asked to study a chemotherapy clinic and to build a simulation model you need detailed data on the nurse's activities. How do you enlist their participation? An authority will rarely be given. You can hope for a sympathetic response or collaborative interest. Another is to somehow offer to the nurses a value that they don't presently have. That may mean an ergonomic study to reduce their fatigue and stress or a custom Excel/Access program that reduces their paperwork. Giving in order to get is probably the best way to generate interest and overcome a "resistance to change."

\section{CONCLUSIONS}

It should be noted the most, but not all, the reported simulation studies in health care are done in a research or consulting framework and that is expected to continue and could accelerate in the US due to the national debate about health care. Much of the current literature, as expected, is accomplished through students and faculty working at colleges and universities (the growth in this section portents even more work). Only a few of these simulations are done by people "inside" health care, although research and consulting collaborators are often "in" the institution. What is not clear is how much simulation is being done, but not reported, in usual course of business inside the health care organizations. It may be that health care organizations simply don't have the resources to conduct their own simulation studies or that the dominant decision makers in a health care organization are unwilling to expend their resources on such analysis.

However with the growth of lean/six sigma organizations within healthcare in the form of performance and operations improvement, new opportunities for wider spread simulation exist. If simulation can become an arm of these efforts, then the opportunity exists for simulation to be augmented by interest in areas of health care delivery where simulation is limited. Quality of care is a major topic addressed in 
lean/six sigma organizations with tools unrelated to simulation, but these could be supported and enhanced with simulation studies. In particular, if the simulation is explained in quality of care terms, it is viewed as more than productivity and more directly addressing patient care.

Simulation needs to be viewed as a means to enhance patient care. It needs to break the mystique of the iron triangle and be viewed as a means to improve cost, quality, and access.

\section{REFERENCES}

AppliedMaterialsInc. 2011. "AUTOMOD." Accessed July 30. www.appliedmaterials.com/servicessoftware/library/applied-automod.

Brailsford, S. C., P. R. Harper, B. Patel, B. and M. Pitt. 2009. "An Analysis of the Academic Literature on Simulation and Modelling in Health Care." J of Simulation 3(3):130-140.

England, W., and S. D. Roberts. 1978. "Applications of Computer Simulation in Health Care." In Proceedings of the 1978 Winter Simulation Conference, edited by H. J. Highland, N. R. Nielsen, and L. G. Hull, 665-676. Piscataway, NJ: Institute of Electrical and Electronic Engineers Inc.

FlexsimSoftwareProducts. 2011. "Flexsim Healthcare - Overview." Accessed July 30. www.flexsim.com/products/healthcare/.

Fone, D., S. Hollinghurst, M. Temple, A. Round, N. Lester, A. Weightman, K. Roberts, E. Coyle, G. Bevan, and S. Palmer. 2003. "Systematic Review of the Use and Value of Computer Simulation Modelling in Population Health and Health Care Delivery." Journal of Public Health 25(4):325-335.

ImagineThat!Inc. 2011. "extendsim: Power Tools for Simulation." Accessed July 30, 2011. www.extendsim.com.

Jacobson, S. H., S. N. Hall, and J. R. Swisher. 2006, "Discrete-Event Simulation of Health Care Systems," Chapter 8 in Patient Flow: Reducing Delay in Healthcare Delivery, edited by R.W. Hall, 211252. Springer International Series.

Jun, J. B., S. H. Jacobson, and J. R. Swisher (1999). "Application of discrete-event simulation in health care clinics: A survey." Journal of the Operational Research Society 50(2): 109-123.

Klein, R. W., R. S. Dittus, S. D. Roberts, and J. R. Wilson. 1993. "Simulation Modeling and Health-Care Decision Making." Medical Decision Making: An International Journal of the Society for Medical Decision Making 13(4):347-354.

Lanner. 2011. "Witness - Simulation for Professional Users." Accessed July 30. www.lanner.com/en/media/witness/witness-pwe.cfm.

Lowery, J. C. 1996. "Introduction to Simulation in Health Care." In Proceedings of the 1996 Winter Simulation Conference, edited by J. M. Charnes, D. J. Morrice, D. T. Brunner, and J. J. Swain, 78-84. Piscataway, New Jersey: Institute of Electrical and Electronics Engineers, Inc.

Lowery, J. C. 1998. "Getting Started in Simulation Healthcare." In Proceedings of the 1998 Winter Simulation Conference, edited by D. J. Medeiros, E. F. Watson, J. S. Carson, and M. S. Manivannan, 3135. Piscataway, New Jersey: Institute of Electrical and Electronics Engineers, Inc.

Oracle. 2011. "Oracle and Crystal Ball." Accessed July 30. www.oracle.com/us/products/applications/crystalball/index.html.

PalisadeCorporation. 2011. "@RISK : a new standard in risk analysis." Accessed July 30. http://www.palisade.com/risk/.

ProModelCorporation. 2011a. "ProModel." Accessed July 30. www.promodel.com/.

ProModelCorporation. 2011b. "MedModel - The Industry for Healthcare Simulations." Accessed July 30, 2011. www.promodel.com/products/medmodel/.

RockwellAutomation. 2011a. "Health Care Simulation." Accessed July 30, 2011. arenasimulation.com/Solutions_Health_Care.aspx.

RockwellSoftware. 2011b. "Arena Simulation Software." Accessed July 30, 2011. www.arenasimulation.com.

SimioLlC. 2011a. "Healthcare Simulation Software." Accessed July 30. www.simio.com/applications/healthcare-simulation-software/healthcare-simulation-software.htm. 
SimioLLC. 2011b. "Simio: Forward Thinking." Accessed July 30. www.simio.com/index.html. Simul8Corporation. 2011a. "Simulation in Healthcare." Accessed July 30. www.simul8.com/healthcare. Simul8Corporation. 2011b. "Simulation Software : 'Visualize, evaluate, optimize, improve.'" Accessed July 30. www.simul8.com.

Standridge, C. R. 1999. "A Tutorial on Simulation in Health Care: Applications and Issues." Proceedings of the 1999 Winter Simulation Conference, IEEE Computer Society. 1: 49-55 vol.41.

Wilson, J. C. T. 1981. "Implementation of Computer Simulation Projects in Health Care." The Journal of the Operational Research Society 32(9):825-832.

WolverineSoftware. 2011a. "GPSS/H - Serving the simulation community since 1977." Accessed July 30. www.wolverinesoftware.com/.

WolverineSoftware. 2011b. "SLX - A new approach to simulation modeling." Accessed July 30. www.wolverinesoftware.com/.

\section{AUTHOR BIOGRAPHIES}

STEPHEN D. ROBERTS is the A. Doug Allison Distinguished Professor in the Edward P. Fitts Department of Industrial and Systems Engineering at North Carolina State University. His research interests include simulation modeling methodology and the simulation modeling of medical and health care decisions. His email address is roberts@ncsu.edu. 